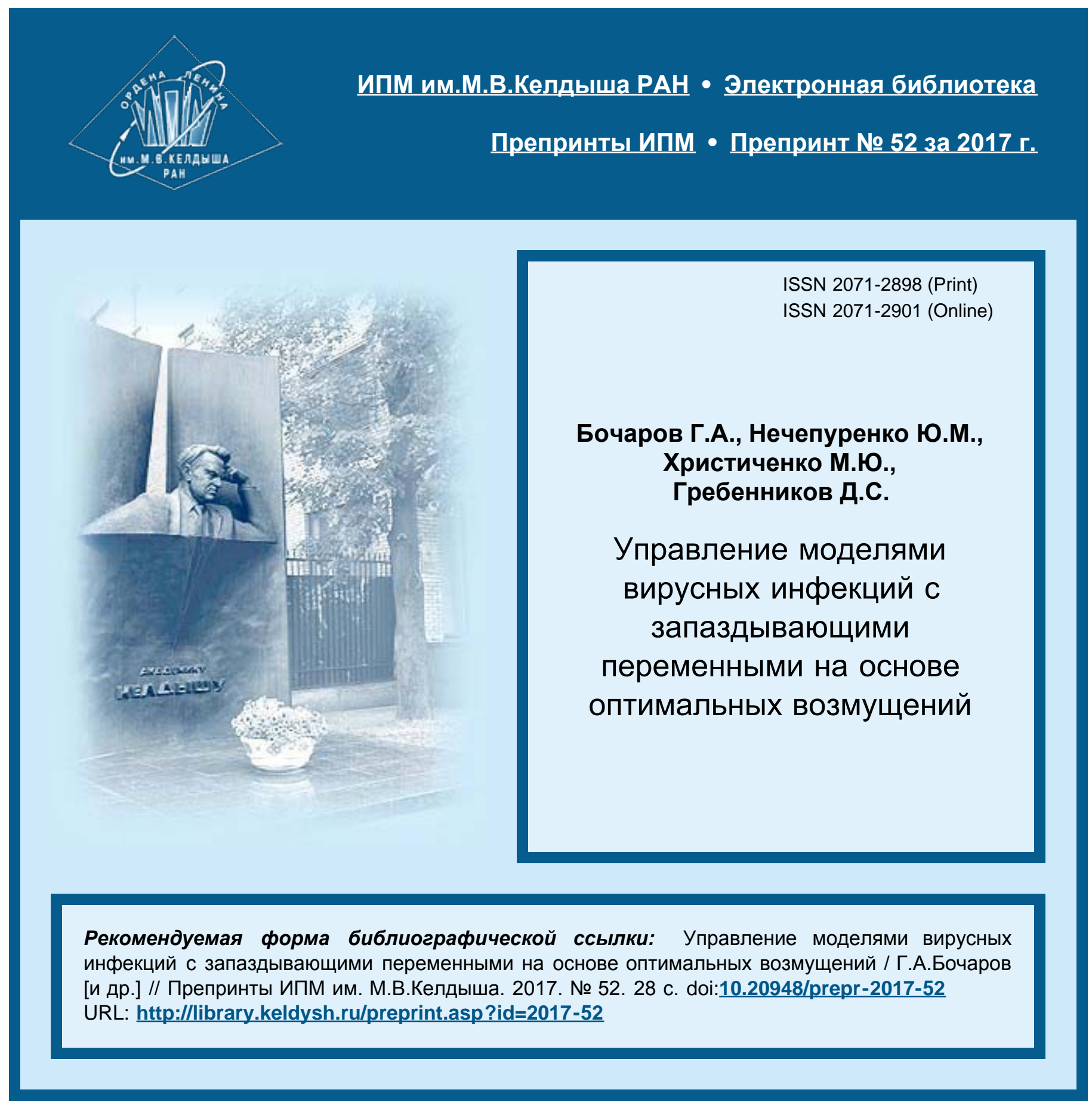




\section{Ордена Ленина ИНСТИТУТ ПРИКЛАДНОЙ МАТЕМАТИКИ имени. М. В. КЕЛДЫША Российской академии наук}

Г. А. Бочаров, Ю. М. Нечепуренко, М. Ю. Христиченко, Д. С. Гребенников

Управление моделями вирусных инфекций с запаздывающими переменными на основе оптимальных возмущений

MOCKBA, 2017 г. 
Г. А. Бочаров, Ю. М. Нечепуренко, М. Ю. Христиченко, Д. С. Гребенников

\section{Управление моделями вирусных инфекций с запаздывающими переменными на основе оптимальных возмущений}

Аннотация. Предложен новый подход к построению мультимодальных воздействий на иммунную систему в хронической фазе вирусной инфекции в рамках математических моделей с запаздывающим аргументом. В качестве возмущений состояния системы предложено использовать так называемые оптимальные возмущения, максимизирующие отклик системы и широко применяемые в теории аэродинамической устойчивости в рамках моделей без запаздывания. Понятие оптимального возмущения перенесено на системы с запаздыванием. Предложен алгоритм вычисления оптимальных возмущений для таких систем. Разработанная технология опробована на системе четырех нелинейных дифференциальных уравнений с запаздывающим временем, представляющей собой модель экспериментальной инфекции, вызванной вирусами лимфоцитарного хориоменингита. С помощью предложенного алгоритма для устойчивых стационарных состояний модели рассчитаны возмущения, характеризующиеся максимальным откликом. Рассмотрены два типа стационарных состояний: с низким и высоким уровнем вирусной нагрузки. Показана возможность коррекции динамики инфекции и восстановления функции специфического звена иммунной системы путем возмущения стационарных состояний.

Ключевые слова: вирусная инфекция, мультимодальное возмущение, математическая модель, дифференциальные уравнения с запаздыванием, стационарное состояние, возмущение, иммунный ответ, оптимальное возмущение

Работа выполнена при финансовой поддержке Российского фонда фундаментальных исследований, проект № 16-01-00572 (разработка и обоснование численных методов), Российского научного фонда, проект № 15-11-00029 (численные эксперименты и биологическая интерпретация их результатов). 
G. A. Bocharov, Yu. M. Nechepurenko, M. Yu. Khristichenko, D. S. Grebennikov

\title{
Control of models of virus infections with delayed variables, based on optimal disturbances
}

\begin{abstract}
A new method for constructing the multi-modal impacts on the immune system in the chronic phase of a viral infection, based on the mathematical models with delayed argument, was proposed. So called, optimal disturbances, widely used in the aerodynamic stability theory with models without delays, were proposed for perturbing the steady states of the system for maximizing the perturbation-induced response. The concept of optimal disturbances was generalized on the systems with delayed argument. An algorithm for computing the optimal disturbances was proposed for such systems. The developed technolo gy was tested using a system of four nonlinear delay-differential equations with delayed time which represents the model of experimental infection in mice caused by lymphocytic choriomeningitis virus. Steady-state perturbations causing the maximum response were computed with the proposed algorithm for two types of steady states: with low and with high level of viral load. The possibility of correction of the infection dynamics and restoration of function of virus specific lymphocytes of immune system by perturbing the steady states was demonstrated.
\end{abstract}

Key words: virus infection, multi-modal perturbation, mathematical model, delay-differential equations, steady state, perturbation, persistence, optimal disturbances 


\section{Содержание}

1. Введение ... . . . . . . . . . . . . . . . . . . . . 5

2. Модель распространения ВЛХМ и ее стационарные состояния . . . . . . . . . . . . . . . . . . . . . 7

3. Оптимальные возмущения стационарных состояний . . . . 10

4. Вычисление оптимальных возмущений . . . . . . . . . . . . . 13

5. Результаты численных экспериментов . . . . . . . . . . . . . 15

6. Заключение . . . . . . . . . . . . . . . . . . . . . . . . . . . 18

Список литературы . . . . . . . . . . . . . . . . 26 


\section{1. Введение}

Динамика вирусных заболеваний человека характеризуется разнообразием течений и исходов, включая острые, хронические и летальные. Хронизация инфекций значительно увеличивает риск развития раковых и аутоиммунных заболеваний, присоединения новых инфекций, поражения сердечнососудистой системы и нервной системы. Проблема изучения закономерностей формирования хронических инфекций и подходов к их лечению была сформулирована как одна из центральных в математической иммунологии в основополагающих работах Г.И. Марчука $[19,18]$. В результате анализа устойчивости стационарных решений базовой модели инфекционного заболевания был предложен подход к выводу организма из хронического состояния путем обострения болезни, т.е. перевода хронической формы в острую. Данный принцип изучения системы путем возмущения её динамики использовался в дальнейшем в подходах к математическому анализу иммунных процессов при различных вирусных заболеваниях, и в первую очередь при инфекции вирусами иммунодефицита человека (ВИЧ), характеризующейся хронической динамикой и летальным исходом [25, 23].

В настоящее время в математической иммунологии широко используются идеи системного анализа, постулированные в области биомедицины как «системная биология» [14]. Предметом исследования системной иммунологии являются динамика, структура и регуляция иммунных процессов. Важнейшим принципом организации иммунной системы, как и любой саморегулируемой биологической системы, является наличие свойства робастности (то есть устойчивости функционирования по отношению к внешним воздействиям) $[9,16]$. Например, ВИЧ инфекцию можно рассматривать как робастную систему «вирус-организм хозяина» [15]. Робастность саморегулируемой системы предполагает свойство хрупкости по отношению к определенной комбинации возмущений $[9,26]$. Поиск соответствующих возмущений параметров или состояния системы, которые позволили бы разработать эффективные режимы терапии, возможен путем построения и анализа свойств чувствительности математической модели заболевания.

Целью данной работы является исследование возможностей построения компенсаторных воздействий на иммунную систему в хронической фазе вирусной инфекции методами математического моделирования. В качестве примера используется разработанная ранее модель экспериментальной инфекции, вызванной вирусами лимфоцитарного хориоменингита (ВЛХМ) [1]. Эта модель, представляющая собой систему 4 нелинейных дифференциальных уравнений с запаздывающим аргументом, кратко описана во втором разделе данной работы.

В качестве возмущений стационарного состояния системы предлага- 
ется использовать так называемые оптимальные возмущения, широко применяемые в теории аэродинамической устойчивости в рамках моделей без запаздывания. В аэродинамике различают естественный (надкритический) и обходной (докритический) сценарии ламинарно-турбулентного перехода [5]. С увеличением числа Рейнольдса при превышении им критического значения, определяющего границу устойчивости к бесконечно малым возмущениям, ламинарное течение заведомо теряет устойчивость (надкритический сценарий). Однако из-за наличия в реальных ламинарных течениях фона конечных возмущений турбулизация таких течений часто происходит при докритических числах Рейнольдса (докритический сценарий). Одним из основных факторов, вызывающих перестройку течения при докритических числах Рейнольдса, является существование в потоке малых возмущений, обладающих способностью значительного роста на конечных временных интервалах. Возмущения, на которых достигается максимально возможный подскок первоначальной энергии, называют оптимальными. Они представляют собой пакеты большого числа ведущих мод со значительно взаимно неортогональными скоростными частями. Для систем с запаздыванием анализ докритических сценариев перестройки решения до настоящего времени никем не проводился. Для таких систем потребовалось сначала выработать физически обоснованные аналоги максимальной амплификацией нормы решения и оптимальных возмущений. Описанию предложенных новых понятий посвящен третий раздел данной работы.

В четвертом разделе предлагается простейший подход к вычислению оптимальных возмущений для систем с запаздыванием. Отметим, что разработанные ранее методы $[4,3,22]$ позволяют эффективно вычислять максимальную амплификацию нормы решения и оптимальные возмущения с заданной точностью для систем обыкновенных дифференциальных уравнений без запаздывания, основываясь на разложении Шура и малоранговой аппроксимации. Эти, а также методы, предложенные в недавней работе [21] для систем с большими разреженными матрицами, представляется целесообразным в будущем перенести на системы с запаздыванием.

Пятый раздел посвящен результатам применения разработанной технологии к упомянутой выше модели инфекции ВЛХМ. С помощью предложенного алгоритма для ее стационарных состояний были найдены возмущения, характеризующиеся максимальным откликом. Рассмотрены два типа стационарных состояний: с низким и высоким уровнем вирусной нагрузки [17]. Первый вариант актуален для задач лечения персистентных вирусных инфекций, характеризующихся численностью вирусов ниже порога обнаружения стандартными методами. Такие субпороговые инфекции являются проблемой при пересадке органов, когда в результате иммуносупрессии происходит обострение соответствующей латентной инфекции. Инфекции с высоким уровнем вирусной нагрузки характерны для ВИЧ, гепатита С и 
В, их лечение также является чрезвычайно актуальной задачей. Поскольку антиретровирусные и иммуномодулирующие препараты обладают побочным действием, минимизация их доз при сохранении уровня отклика системы является важной компонентой разработки стратегии лечения. С учетом этого на примере модели инфекции ВЛХМ была исследована возможность коррекции динамики инфекции и восстановления функции Т-лимфоцитов путем введения малых возмущений, приводящих к максимальному отклику.

В шестом разделе подведен итог данной работы.

\section{2. Модель распространения ВЛХМ и ее ста- ционарные состояния}

Простейшая математическая модель динамики инфекции, вызванной вирусами лимфоцитарного хориоменингита, приведенная и исследованная в работе [17], сформулирована в виде системы нелинейных дифференциальных уравнений с запаздывающим аргументом. Система описывает динамику следующих переменных: концентрации вирусных частиц $V$, двух популяций специфичных к ВЛХМ цитотоксических лимфоцитов (ЦТЛ) клеток-прекурсоров $E_{p}$ и клеток-эффекторов $E_{e}$, а также кумулятивной вирусной нагрузки $W$. Система имеет вид:

$$
\begin{aligned}
\frac{d}{d t} V(t)= & \beta V(t)\left(1-\frac{V(t)}{V_{m v c}}\right)-\gamma_{V E} E_{e}(t) V(t) \\
\frac{d}{d t} E_{p}(t)= & \alpha_{E_{p}}\left(E_{p}^{0}-E_{p}(t)\right)+\beta_{p} g_{p}(W) V(t-\tau) E_{p}(t-\tau) \\
& -\alpha_{A P} V\left(t-\tau_{A}\right) V(t) E_{p}(t) \\
\frac{d}{d t} E_{e}(t)= & b_{d} g_{e}(W) V(t-\tau) E_{p}(t-\tau) \\
& -\alpha_{A E} V\left(t-\tau_{A}\right) V(t) E_{e}(t)-\alpha_{E_{e}} E_{e}(t) \\
\frac{d}{d t} W(t)= & b_{W} V(t)-\alpha_{W} W(t)
\end{aligned}
$$

где $g_{p}(W)=1 /\left(1+W / \theta_{p}\right)^{2}, g_{e}(W)=1 /\left(1+W / \theta_{E}\right)^{2}$. Биологический смысл параметров системы пояснен в Табл. 1.

Для определения решения при $t>0$ достаточно задать значения $V(t)$ при $-\tau_{A} \leq t \leq 0$, значения $E_{p}(t)$ при $-\tau \leq t \leq 0$, значения $E_{e}(0)$ и $W(0)$. Однако для единообразия далее мы будем предполагать, что начальные значения всех переменных заданы при $-\tau_{A} \leq t \leq 0$.

Задача Коши для системы (1) с неотрицательными начальными условиями и неотрицательными параметрами глобально разрешима на любом 
Таблица 1: Биологический смысл параметров модели (1).

\begin{tabular}{|c|l|}
\hline Параметр & Биологические смысл \\
\hline$\beta$ & Константа скорости репликации вирусных частиц \\
$\gamma_{V E}$ & Константа скорости элиминации вирусов \\
$V_{m v c}$ & за счет клеток-эффекторов \\
$\tau$ & Максимально возможная концентрация \\
$b_{p}$ & Характерная продолжительность цикла деления ЦТЛ \\
$b_{d}$ & Константа скорости стимуляции ЦТЛ \\
$\theta_{p}$ & Константа скорости дифференциции ЦТЛ \\
$\theta_{E}$ & Порог вирусной нагрузки для перехода \\
$\alpha_{E_{p}}$ & Порог вирусной нагрузки для перехода \\
$\alpha_{E_{e}}$ & Эффекторов в состояние анергии \\
$E_{p}^{0}$ & Константа скорости естественной гибели прекурсоров \\
$\tau_{A}$ & Концентрация прекурсоров в селезенке мыши, \\
$\alpha_{A P}$ & Хе имевшей контакта с ВЛХМ \\
$\alpha_{A E}$ & Корактерная продолжительность перехода ЦТЛ к апоптозу \\
$b_{W}$ & Константа скорости апоптоза эффекторов \\
$\alpha_{W}$ & Константа скорости роста кумулятивной вирусной нагрузки \\
& Константа скорости восстановления организма \\
& от воздействий вирусной нагрузки \\
\hline
\end{tabular}

конечном интервале времени. Данное утверждение можно доказать, используя технику, описанную в [19], на основе метода шагов Беллмана, рассматривая линейную систему обыкновенных дифференциальных уравнений, мажорирующую правую часть системы уравнений модели.

Обозначив вектор переменных системы (1) через

$$
U(t)=\left(V(t), E_{p}(t), E_{e}(t), W(t)\right)^{T},
$$

будем записывать эту систему в следующем компактном виде:

$$
\frac{d}{d t} U(t)=F\left(U(t), U(t-\tau), U\left(t-\tau_{A}\right)\right) .
$$

В соответствии со сказанным выше будем считать, что вектор переменных $U(t)$ задан при $-\tau_{A} \leq t \leq 0$. 
Модель (3) при различных наборах параметров имеет различные стационарные состояния. В данной работе мы использовали два набора параметров, для каждого из которых было найдено устойчивое стационарное состояние. Стационарные состояния вычислялись путем применения метода Ньютона к нелинейному уравнению $\Phi(U)=0$, где $\Phi(U)=F(U, U, U)$. Поиск наборов параметров, при которых стационарные состояния модели (3) обладают требуемыми свойствами, и соответствующих начальных значений для метода Ньютона был выполнен, основываясь на результатах численного бифуркационного анализа, проведенного в работе [17]. Вариацией параметров в области допустимых значений, указанных в [17], были найдены стационарные состояния, описывающие два варианта хронической инфекции. Первое стационарное состояние соответствует латентной форме инфекции с низким уровнем вирусной нагрузки и большой численностью клеток иммунологической памяти [7]. Второе - описывает клинически выраженную хроническую инфекцию с большой вирусной нагрузкой и частичным истощением популяции Т-лимфоцитов, специфичных к антигенам вируса [20, 8]. Соответственно, целью возмущения системы в первом случае может являться либо активация инфекционного процесса с последующим удалением вирусного резервуара, либо непосредственная нейтрализация инфекции, а во втором - восстановление реактивности истощенного звена иммунной системы и снижение вирусной нагрузки. Данные сценарии динамики процесса имеют место при ВИЧ инфекции и соответствуют фенотипически различным вариантам инфекции, наблюдаемым у «элит-контроллеров» и «прогрессоров» соответственно $[13,11]$.

Значения параметров модели (3), соответствующие указанным двум стационарным состояниям $\bar{U}_{I}$ и $\bar{U}_{I I}$, приведены в Табл. 2. Значения компонент этих стационарных состояний приведены в Табл. 3. 
Таблица 2: Значения параметров модели (1), соответствующие исследуемым стационарным состояниям $\bar{U}_{I}$ и $\bar{U}_{I I}$.

\begin{tabular}{|c|c|ll|}
\hline Параметр & Размерность & $\bar{U}_{I}$ & $\overline{U_{I I}}$ \\
\hline$\beta$ & $1 /$ сутки & 1.2 & 0.08 \\
$E_{p}^{0}$ & клетка/мл & $10^{6}$ & $10^{3}$ \\
$b_{p}$ & мл/(частиц·сутки $)$ & $7.73 \cdot 10^{-5}$ & $1 \cdot 10^{-5}$ \\
$b_{d}$ & мл/(частиц·сутки $)$ & $7.73 \cdot 10^{-4}$ & $5 \cdot 10^{-4}$ \\
$\theta_{p}$ & частицы $/$ мл & $3 \cdot 10^{6}$ & 10 \\
$\theta_{E}$ & частицы $/$ мл & $1 \cdot 10^{5}$ & $1.8 \cdot 10^{6}$ \\
\hline$\gamma_{V E}$ & мл/(клетка·сутки $)$ & $1.34 \cdot 10^{-6}$ \\
$V_{m v c}$ & частицы $/$ мл & $4.82 \cdot 10^{7}$ \\
$\alpha_{E_{p}}$ & $1 /$ сутки & 0.5 \\
$\alpha_{E_{e}}$ & $1 /$ сутки & 0.1 \\
$\tau$ & сутки & 0.4 \\
$\tau_{A}$ & сутки & 5.6 \\
$\alpha_{A P}$ & (мл/частицы $)^{2} /$ сутки & $7.5 \cdot 10^{-16}$ \\
$\alpha_{A E}$ & (мл/частицы $)^{2} /$ сутки & $4.36 \cdot 10^{-14}$ \\
$b_{W}$ & $1 /$ сутки & 1 \\
$\alpha_{W}$ & $1 /$ сутки & 0.11 \\
\hline
\end{tabular}

\section{3. Оптимальные возмущения стационарных со- стояний}

Нас будет интересовать поведение системы (3) вблизи устойчивого стационарного состояния. Записывая решение вблизи стационарного состояния в виде $U(t)=\bar{U}+\varepsilon U^{\prime}(t)+O\left(\varepsilon^{2}\right)$, где $\varepsilon-$ малый по абсолютной величине параметр, подставляя это решение в (3) и требуя, чтобы полученные равенства выполнялись при всех сколь угодно малых значениях $\varepsilon$, для функции $U^{\prime}(t)$ получим следующую систему линейных дифференциальных уравнений:

$$
\frac{d}{d t} U^{\prime}(t)=L_{0} U^{\prime}(t)+L_{\tau} U^{\prime}(t-\tau)+L_{\tau_{A}} U^{\prime}\left(t-\tau_{A}\right),
$$

где

$$
L_{0}=\left(\begin{array}{cccc}
\beta-\frac{2 \beta \bar{V}}{V_{v m c}}-\gamma_{V E} \overline{E_{e}} & 0 & -\gamma_{V E} \bar{V} & 0 \\
-\alpha_{A P} \bar{V} \overline{E_{p}} & -\alpha_{E_{p}}-\alpha_{A P} \bar{V}^{2} & 0 & \frac{-2 b_{p} \bar{V} \overline{E_{p}} g_{p}(\bar{W})}{\theta_{p}+\bar{W}} \\
-\alpha_{A E} \bar{V} \overline{E_{e}} & 0 & -\alpha_{E_{e}}-\alpha_{A E} \bar{V}^{2} & \frac{-2 b_{d} \bar{V} \overline{E_{p}} g_{e}(\bar{W})}{\theta_{E}+\bar{W}} \\
b_{W} & 0 & 0 & -\alpha_{W}
\end{array}\right),
$$


Таблица 3: Округленные до трех значащих десятичных цифр значения компонент стационарного состояния и максимальные и минимальные значения компонент возмущенного стационарного состояния, соответствующие Рис.1 $-6$.

\begin{tabular}{|c|c|c|c|c|c|}
\hline & & $V$ & $E_{p}$ & $E_{e}$ & $W$ \\
\hline \multicolumn{2}{|c|}{ Состояние $\bar{U}_{I}$} & 11.5 & $1.01 \cdot 10^{6}$ & $8.96 \cdot 10^{5}$ & 104 \\
\hline \multirow{4}{*}{ Рис.1 } & $\min$ & $2.75 \cdot 10^{-10}$ & 974 & $3.98 \cdot 10^{5}$ & $5.49 \cdot 10^{-3}$ \\
\hline & $t_{\min }$ & 69.6 & -0.92 & $-10^{-3}$ & 123 \\
\hline & $\max$ & 330 & $1.08 \cdot 10^{6}$ & $1.58 \cdot 10^{6}$ & $1.12 \cdot 10^{3}$ \\
\hline & $t_{\max }$ & 6.63 & 10.3 & 11.9 & 9.13 \\
\hline \multirow{4}{*}{ Рис.2 } & $\min$ & $1.64 \cdot 10^{-23}$ & $9.6 \cdot 10^{5}$ & $1.94 \cdot 10^{5}$ & $8.02 \cdot 10^{-6}$ \\
\hline & $t_{\min }$ & 92 & -0.46 & -0.46 & 184 \\
\hline & $\max$ & 700 & $1.13 \cdot 10^{6}$ & $2.03 \cdot 10^{6}$ & $1.82 \cdot 10^{3}$ \\
\hline & $t_{\max }$ & 5.55 & 8.45 & 9.58 & 7.46 \\
\hline \multirow{4}{*}{ Рис.3 } & $\min$ & $5.38 \cdot 10^{-6}$ & $10^{6}$ & $5.37 \cdot 10^{5}$ & $2.59 \cdot 10^{-2}$ \\
\hline & $t_{\min }$ & 45.6 & 88.2 & 98.9 & 79.8 \\
\hline & $\max$ & 174 & $1.06 \cdot 10^{6}$ & $1.39 \cdot 10^{6}$ & 703 \\
\hline & $t_{\max }$ & 107 & 111 & $-10^{-3}$ & 110 \\
\hline \multirow{4}{*}{ Рис.4 } & $\min$ & $9.44 \cdot 10^{-11}$ & $10^{6}$ & $4.4 \cdot 10^{5}$ & $7.48 \cdot 10^{-4}$ \\
\hline & $t_{\min }$ & 58.9 & 119 & 132 & 111 \\
\hline & $\max$ & 272 & $1.08 \cdot 10^{6}$ & $1.6 \cdot 10^{6}$ & 947 \\
\hline & $t_{\max }$ & 139 & 143 & -0.46 & 142 \\
\hline \multicolumn{2}{|c|}{ Состояние $\bar{U}_{I I}$} & $1.35 \cdot 10^{5}$ & $10^{3}$ & $5.95 \cdot 10^{4}$ & $1.23 \cdot 10^{6}$ \\
\hline \multirow{4}{*}{ Рис.5 } & $\min$ & $6.4 \cdot 10^{3}$ & $10^{3}$ & $9.45 \cdot 10^{3}$ & $1.8 \cdot 10^{5}$ \\
\hline & $t_{\min }$ & 8.92 & -5.6 & 17.9 & 28.4 \\
\hline & $\max$ & $1.35 \cdot 10^{5}$ & $3.14 \cdot 10^{4}$ & $6.5 \cdot 10^{5}$ & $1.23 \cdot 10^{6}$ \\
\hline & $t_{\max }$ & -5.6 & $-10^{-3}$ & 1.57 & -5.6 \\
\hline \multirow{4}{*}{ Рис.6 } & $\min$ & 3.96 & $10^{3}$ & 25.4 & $2.34 \cdot 10^{3}$ \\
\hline & $t_{\min }$ & 10.7 & -5.6 & 34.4 & 65.4 \\
\hline & $\max$ & $1.35 \cdot 10^{5}$ & $1.53 \cdot 10^{5}$ & $2.45 \cdot 10^{6}$ & $1.23 \cdot 10^{6}$ \\
\hline & $t_{\max }$ & -5.6 & $-10^{-3}$ & 1.08 & -5.6 \\
\hline
\end{tabular}

$$
L_{\tau}=\left(\begin{array}{cccc}
0 & 0 & 0 & 0 \\
b_{p} \overline{E_{p}} g_{p}(\bar{W}) & b_{p} \bar{V} g_{p}(\bar{W}) & 0 & 0 \\
b_{d} \overline{E_{p}} g_{e}(\bar{W}) & b_{d} \bar{V} g_{e}(\bar{W}) & 0 & 0 \\
0 & 0 & 0 & 0
\end{array}\right), \quad L_{\tau_{A}}=\left(\begin{array}{cccc}
0 & 0 & 0 & 0 \\
-\alpha_{A P} \bar{V} \overline{E_{p}} & 0 & 0 & 0 \\
-\alpha_{A E} \bar{V} \overline{E_{e}} & 0 & 0 & 0 \\
0 & 0 & 0 & 0
\end{array}\right)
$$

Систему (4) будем называть линеаризованными уравнениями эволю- 
ции возмущений. Начальные значения этой системы будем задавать, как и начальные значения системы (3), в интервале $-\tau_{A} \leq t \leq 0$.

Для вектора переменных системы (4) введем в рассмотрение следующее семейство локальных норм в момент времени $t$ :

$$
\left\|U^{\prime}\right\|_{D, t}=\left(\int_{t-\tau_{A}}^{t}\left\|D U^{\prime}(\xi)\right\|_{2}^{2} d \xi\right)^{1 / 2}
$$

где $D$ - заданная положительно определенная диагональная матрица, а $\|\cdot\|_{2}$ - вторая (евклидова) векторная норма.

Под оптимальным возмущением $U_{o p t}^{\prime}(t)$ будем понимать решение системы (4), обеспечивающее максимальный подскок значения локальной нормы по сравнению с первоначальным. То есть такое возмущение $U^{\prime}(t)$, при котором достигается максимум величины

$$
\max _{t \geq 0} \frac{\left\|U^{\prime}\right\|_{D, t}}{\left\|U^{\prime}\right\|_{D, 0}} .
$$

Поскольку оптимальное возмущение по определению является некоторым решением линейной системы (4) и, следовательно, полностью определяется своим значением в интервале $-\tau_{A} \leq t \leq 0$, при построении оптимального возмущения наряду с выбором нормы, в которой проводится оптимизация, принципиальным является вопрос о том, из какого пространства функций, заданных в интервале $-\tau_{A} \leq t \leq 0$, мы берем начальные значения. Это подпространство функций $\left[-\tau_{A}, 0\right] \rightarrow \mathbb{R}^{4}$ мы далее будем обозначать через $\mathcal{Q}$. Для существования максимума необходимо, чтобы $\mathcal{Q}$ было полным пространством в норме $\|\cdot\|_{D, 0}$. На практике достаточно выбирать в качестве $\mathcal{Q}$ линейную оболочку какого-либо конечного набора базисных функций. Это, в частности, гарантирует его полноту.

Искать оптимальное возмущение удобнее в два этапа. Сначала вычисляем максимальную амплификацию

$$
\Gamma(t)=\max _{U^{\prime}} \frac{\left\|U^{\prime}\right\|_{D, t}}{\left\|U^{\prime}\right\|_{D, 0}}
$$

локальной нормы решения системы (4), где максимум берется по всем решениям, начальные значения которых ненулевые и принадлежат $\mathcal{Q}$, и находим $t=t_{\text {opt }}$, при котором функция $\Gamma(t)$ достигает максимального значения. Если таких $t$ несколько, то для определенности берем из них минимальное. Таким образом,

$$
t_{\text {opt }}=\min \arg \max _{t \geq 0} \Gamma(t)
$$


Затем находим

$$
U_{o p t}^{\prime} \in \arg \max _{U^{\prime}} \frac{\left\|U^{\prime}\right\|_{D, t_{o p t}}}{\left\|U^{\prime}\right\|_{D, 0}} .
$$

Если $D$ и $\mathcal{Q}$ фиксированы, то любое найденное оптимальное возмущение обеспечивает один и тот же максимальный подскок локальной нормы решения. Обычно максимальная амплификация имеет только одну точку максимума, а решение второй оптимизационной задачи однозначно с точностью до ненулевой мультипликативной константы.

Найденное оптимальное возмущение мы будем использовать для возмущения стационарных состояний исходной нелинейной модели (3). Для этого в качестве начальных значений мы будем брать

$$
U(t)=\bar{U}+\varepsilon \widetilde{U}_{o p t}^{\prime}(t)
$$

при $-\tau_{A} \leq t \leq 0$, где $\widetilde{U}_{\text {opt }}^{\prime}(t)$ означает нормированное оптимальное возмущение, а $\varepsilon$ - параметр. Варьируя этот параметр, можно увеличивать или уменьшать начальное возмущение стационарного состояния. Если абсолютная величина $\varepsilon$ мала, то следует ожидать, что полученное решение $U(t)$ системы (3) будет близко к (7) при $t>0$. При больших по абсолютной величине $\varepsilon$ из-за влияния нелинейности решение системы (3) будет значительно отличаться от $(7)$ при $t>0$. Существенную роль играет и знак $\varepsilon$. В зависимости от него плотность популяции вируса при $t>0$ либо начинает нарастать, либо убывать. Если оптимальное возмущение в (7) нормировано так, что первая компонента вектора

$$
L_{0} \widetilde{U}_{o p t}^{\prime}(0)+L_{\tau} \widetilde{U}_{o p t}^{\prime}(-\tau)+L_{\tau_{A}} \widetilde{U}_{o p t}^{\prime}\left(-\tau_{A}\right)
$$

является положительной, то плотность популяции вируса нарастает при $t>$ 0 в случае $\varepsilon>0$.

\section{4. Вычисление оптимальных возмущений}

Оптимальные возмущения можно вычислять на основе любой разностной схемы, подходящей для решения задач Коши для систем линейных обыкновенных дифференциальных уравнений с запаздывающим аргументом. В данной работе мы использовали неявную схему второго порядка BDF2 [12] на равномерной сетке

$$
\left\{t_{k}=\delta k: k=-m_{A}+1,-m_{A}+2, \ldots\right\},
$$

построенной в полуинтервале $\left(-\tau_{A}, \infty\right)$ с шагом $\delta>0$. Величины $m=[\tau / \delta]$ и $m_{A}=\left[\tau_{A} / \delta\right]$, где [.] означает целую часть числа, являлись дискретными 
аналогами задержек $\tau$ и $\tau_{A}$ соответственно. После дискретизации описанным выше способом система (4) примет вид

$$
\begin{array}{r}
\frac{1.5 U_{k}-2 U_{k-1}+0.5 U_{k-2}}{\delta}=L_{0} U_{k}+L_{\tau} U_{k-m}+L_{\tau_{A}} U_{k-m_{A}}, \\
k=1,2, \ldots,
\end{array}
$$

где $U_{k}$ - сеточная функция, аппроксимирующая $U\left(t_{k}\right)$. В качестве начальных данных для решения задачи Коши нужно задать значения $U_{-m_{A}+1}, \ldots, U_{0}$.

Запишем уравнение (8) в виде

$$
U_{k}=C_{1} U_{k-1}+C_{2} U_{k-2}+C_{m} U_{k-m}+C_{m_{A}} U_{k-m_{A}},
$$

где

$$
\begin{gathered}
C_{1}=2\left(1.5 I-\delta L_{0}\right)^{-1}, \quad C_{2}=-0.5\left(1.5 I-\delta L_{0}\right)^{-1}, \\
C_{m}=\left(1.5 I-\delta L_{0}\right)^{-1} \delta L_{\tau}, \quad C_{m_{A}}=\left(1.5 I-\delta L_{0}\right)^{-1} \delta L_{\tau_{A}},
\end{gathered}
$$

a $I$ означает единичную матрицу четвертого порядка, и дополним уравнение (9) тождествами $U_{j}=U_{j}, j=k-1, \ldots, k-m_{A}+1$. Полученную таким образом систему из $m_{A}$ уравнений можно записать в виде

$$
X_{k}=M X_{k-1},
$$

где

$$
X_{k}=\left(\begin{array}{c}
U_{k} \\
\vdots \\
U_{k-m_{A}+1}
\end{array}\right), \quad M=\left(\begin{array}{ccc}
M_{11} & \cdots & M_{1 m_{A}} \\
\vdots & & \vdots \\
M_{m_{A} 1} & \cdots & M_{m_{A} m_{A}}
\end{array}\right) .
$$

Матрица $M$ в (11) является блочной, блочного порядка $m_{A}$ с блоками порядка 4. Все блоки этой матрицы нулевые, кроме поддиагональных блоков $M_{j+1, j}=I\left(j=1, \ldots, m_{A}-1\right)$ и четырех блоков $M_{11}=C_{1}, M_{12}=C_{2}$, $M_{1 m}=C_{m}$ и $M_{1 m_{A}}=C_{m_{A}}$, стоящих в первой блочной строке.

В силу (10), (11) сеточный аналог $\Gamma_{k}$ максимальной амплификации (6) нормы решения можно записать следующим образом:

$$
\Gamma_{k}=\max _{X_{0} \in \operatorname{span}(Q) \backslash\{0\}} \frac{\left\|H M^{k} X_{0}\right\|_{2}}{\left\|H X_{0}\right\|_{2}},
$$

где $Q$ - матрица размера $4 m_{A} \times p\left(p \leq 4 m_{A}\right)$, столбцы которой образуют базис в сеточном аналоге подпространства $\mathcal{Q}, \operatorname{span}(Q)$ означает линейную оболочку столбцов матрицы $Q, H=I_{m_{A}} \otimes D, D$ - диагональная матрица, задающая локальную норму, в которой вычисляется оптимальное возмущение, а $\otimes-$ символ Кронекерова произведения. 
Учитывая, что

$$
\Gamma_{k}=\left\|H M^{k} H^{-1} \widetilde{Q}\right\|_{2}
$$

где $\widetilde{Q}$ - матрица, полученная ортогонализацией столбцов матрицы $H Q$, вычисление $\Gamma_{k}$ сводится к формированию матрицы $Y_{0}=H^{-1} \widetilde{Q}$ и вычислению матриц $Y_{k}$ по рекуррентной формуле $Y_{k}=M Y_{k-1}$ с одновременным вычислением норм матриц $H Y_{k}$.

Пусть $k_{\text {opt }}$ означает значение $k$, при котором достигается максимум $\Gamma_{k}$. Вычислив нормированный правый сингулярный вектор $\eta$ матрицы

$$
H M^{k_{o p t}} H^{-1} \widetilde{Q}
$$

отвечающий ее максимальному сингулярному числу [10], начальное значение $X_{0}^{o p t}$ сеточного аналога оптимального возмущения $X_{k}^{\text {opt }}$ можно найти по формуле $X_{0}^{o p t}=H^{-1} \widetilde{Q} \eta$.

Отметим, что для повышения эффективности описанного выше алгоритма матрицу $M$ надо хранить и умножать на векторы в разреженном формате.

\section{5. Результаты численных экспериментов}

Оптимальные возмущения искались в подпространстве $\mathcal{Q}$ функций, кусочно-постоянных в интервале $\left[-\tau_{A}, 0\right]$ с $l$ одинаковыми по длине подынтервалами постоянности. Для расчета оптимальных возмущений использовалась сетка с шагом $\delta=10^{-2}$. Всего было рассчитано и использовано два оптимальных возмущения (с $l=6$ и 12 ) для стационарного состояния $\bar{U}_{I}$ и одно $($ с $l=6)$ для стационарного состояния $\bar{U}_{I I}$. В качестве весов нормы (диагональные элементы матрицы $D$ ) для первых двух возмущений были использованы величины, обратные к компонентам вектора $\bar{U}_{I}$. При расчете третьего оптимального возмущения в качестве подпространства начальных значений $\mathcal{Q}$ выбиралось подпространство функций, у которых компоненты $V$ и $W$ являются тождественными нулями. Веса нормы выбирались единичными. Во всех трех случаях максимальная амплификация, т.е. максимальное сингулярное число $\Gamma\left(t_{\text {opt }}\right)$ матрицы $(12)$, и второе максимальное сингулярное число $\widetilde{\Gamma}\left(t_{\text {opt }}\right)$ этой матрицы были очень хорошо отделены друг от друга (см. Табл. 4), что свидетельствовало о единственности оптимального возмущения с точностью до ненулевой мультипликативной константы.

Для интегрирования системы (3) с начальными данными (7) шаг сетки выбирался равным $10^{-3}$ и использовалась та же схема BDF2, что и при вычислении оптимальных возмущений. Оптимальные возмущения интерполировались на более мелкую сетку стандартным алгоритмом кубической интерполяции, сохраняющим монотонность и выпуклость. Параметр $\varepsilon$ выби- 
Таблица 4: Результаты расчета максимальной амплификации.

\begin{tabular}{|c|c|c|c|c|}
\hline Состояние & $l$ & $t_{\text {opt }}$ & $\Gamma\left(t_{\text {opt }}\right)$ & $\widetilde{\Gamma}\left(t_{\text {opt }}\right)$ \\
\hline $\bar{U}_{I}$ & 6 & 17.0 & $2.98 \cdot 10^{2}$ & 2.72 \\
\hline $\bar{U}_{I}$ & 12 & 17.0 & $4.19 \cdot 10^{2}$ & 3.83 \\
\hline $\bar{U}_{I I}$ & 6 & 23.5 & $3.16 \cdot 10^{2}$ & $5.57 \cdot 10^{-2}$ \\
\hline
\end{tabular}

рался по абсолютной величине таким, чтобы получить достаточно большой отклик при малом начальном возмущении. Отметим, что для численного интегрирования системы (1) ее авторы использовали код DIFSUB-DDE, предназначенный для численного решения жестких систем нелинейных дифференциальных уравнений с постоянными запаздывающими аргументами [2]. В коде реализована модификация линейного многошагового метода Гира по схеме BDF6 переменного порядка $p \leq 6$ с обработкой точек разрыва производных до $p+1$ порядка, для аппроксимации запаздывающих компонент используется интерполяционный вектор Нордсика. С помощью этого кода были проведены верификационные расчеты, показавшие хорошее совпадение с результатами расчетов с помощью BDF2 метода.

Начальные значения возмущенного стационарного состояния $\bar{U}_{I}$ и результаты их интегрирования приведены на Рис. $1-4$, где красные линии соответствуют стационарному состоянию, а синие - возмущенному стационарному состоянию. В верхней части Табл. 3 приведены округленные до трех значащих десятичных цифр максимальные и минимальные значения компонент возмущенного стационарного состояния, соответствующие этим рисункам.

Рис. 1 демонстрирует развитие инфекционного процесса в результате возмущения начального стационарного состояния путем увеличения вирусной нагрузки, кумулятивной вирусной нагрузки и снижения численности Т-лимфоцитов. Рассматривается состояние с низкой вирусной нагрузкой. Целью возмущения системы является активация инфекционного процесса путем его обострения, с последующей элиминацией. Как видно из рисунка, оптимальное возмущение позволяет достичь эту цель. Оно приводит к существенному увеличению (на несколько порядков) вирусной нагрузки и последующему выведению вирусной популяции (см. Табл. 3). Данный сценарий возмущений соответствует лечебными воздействиям, временно (в течение 1 суток) подавляющим иммунитет и активирующим репликацию вирусов.

Рис. 2 демонстрирует эффект уменьшения длительности возмущения до 0.5 суток и усложнения характера возмущения вирусной нагрузки: комбинацию снижения и последующего увеличения вирусной нагрузки, увеличения кумулятивной вирусной нагрузки и уменьшения численности Т- 
лимфоцитов. Видно, что оптимальное возмущение данного типа также приводит к достижению цели воздействия - существенному увеличению (на несколько порядков) вирусной нагрузки, незначительному влиянию на численность специфических Т-клеток, и завершается снижением вирусной нагрузки до уровня, соответствующего полной элиминации. Данный сценарий возмущений соответствует гипотетическим структурированным лечебным воздействиям, временно снижающим численность вирусов, а затем увеличивающим вирусную популяцию и подавляющим специфические реакции.

Рис. 3 демонстрирует развитие инфекционного процесса в результате возмущения начального стационарного состояния путем снижения вирусной нагрузки, кумулятивной вирусной нагрузки и увеличения численности Тлимфоцитов. Целью возмущения системы в состоянии с низкой вирусной нагрузкой в данном случае является элиминация инфекции без её обострения. Время действия возмущения 1 сутки. Видно, что оптимальное возмущение данного типа приводит к достижению цели воздействия - существенному снижению вирусной нагрузки до уровня, соответствующего полной нейтрализации инфекции. Данный сценарий возмущений соответствует лечебным воздействиям, временно (в течение 1 суток) нейтрализующим часть вирусов и увеличивающим уровень специфических Т-клеток за счет их адоптивного переноса.

На Рис. 4 исследовано влияние структурированного лечебного воздействия на развитие инфекционного процесса в результате возмущения начального стационарного состояния путем комбинации увеличения и последующего снижения вирусной нагрузки, снижения кумулятивной вирусной нагрузки и увеличения численности Т-лимфоцитов. Целью возмущения системы является элиминация вирусной популяции. Время действия возмущения в два раза короче, чем в предыдущем случае. Видно, что оптимальное возмущение данного типа приводит к достижению цели воздействия - существенному снижению (на несколько порядков) вирусной нагрузки и незначительному влиянию на уровень специфических Т-клеток (см. Табл. 3). Данный сценарий возмущений соответствует лечебным воздействиям, приводящим к нейтрализации вирусов персистирующих ниже порога обнаружения без активации инфекционного процесса.

Начальные значения возмущенного стационарного состояния $\bar{U}_{I I}$ и результаты их интегрирования приведены на Рис. 5 - 6. В нижней части Табл. 3 приведены округленные до трех значащих десятичных цифр максимальные и минимальные значения компонент возмущенного стационарного состояния, соответствующие этим рисункам. Оба рисунка демонстрируют развитие инфекционного процесса в результате возмущения начального стационарного состояния с высоким уровнем вирусной нагрузки. Целью исследования был поиск оптимального возмущения, которое приводит к увеличению 
специфического иммунитета и снижению вирусной нагрузки. Длительность возмущения - 1 сутки.

Структура возмущения, представленная на Рис. 5 (при неизменных значениях вирусной и кумулятивной вирусной нагрузок увеличена численность наивных Т-лимфоцитов на порядок, в сочетании с незначительным увеличением числа Т-клеток эффекторов 1\%), приводит к развитию более сильного иммунного ответа (увеличению на порядок числа Т-лимфоцитовэффекторов) и 20 кратному снижению численности вирусов, чего, однако, недостаточно для освобождения организма от инфекции. Рис. 6 демонстрирует развитие инфекционного процесса при пятикратном увеличении возмущения, что приводит к иммунному ответу, достаточному для снижения вирусной нагрузки примерно в 4000 раз. Это можно интерпретировать как практическую элиминацию вирусов из организма. Более точные характеристики решения представлены в Табл. 3. Таким образом, показано существование оптимальных возмущений, приводящих к переходному процессу с большой амплитудой вариации решения, прежде всего - вирусной переменной, что и являлось главной целью воздействия на систему.

\section{6. Заключение}

В данной работе проведено исследование отклика математической модели экспериментальной инфекции вирусами лимфоцитарного хориоменингита на мультимодальные воздействия. Целью работы было построение начальных возмущений для системы дифференциальных уравнений с запаздывающим аргументом, для которых реакция динамической системы, т.е. отклонение решения от стационарного, является максимальной. Эта проблема является ключевой для системной иммунологии, поскольку её решение позволит строить более эффективные схемы многокомпонентной терапии вирусных инфекций, в частности ВИЧ инфекции [6].

Впервые для задач математической иммунологии был реализован алгоритм построения возмущения начального состояния системы с помощью так называемых оптимальных возмущений, обеспечивающих максимальную амплификацию возмущения в заданной норме. Для двух типов устойчивых стационарных состояний модели, отвечающих биологическим сценариям (I) персистенции вирусов ниже порога обнаружения и (II) хроническим вирусным инфекциям с высоким уровнем вирусной нагрузки и истощением Т-лифмоцитов, были рассчитаны несколько структурно различных типов возмущений и исследовано их влияние на динамику инфекционного процесca. 

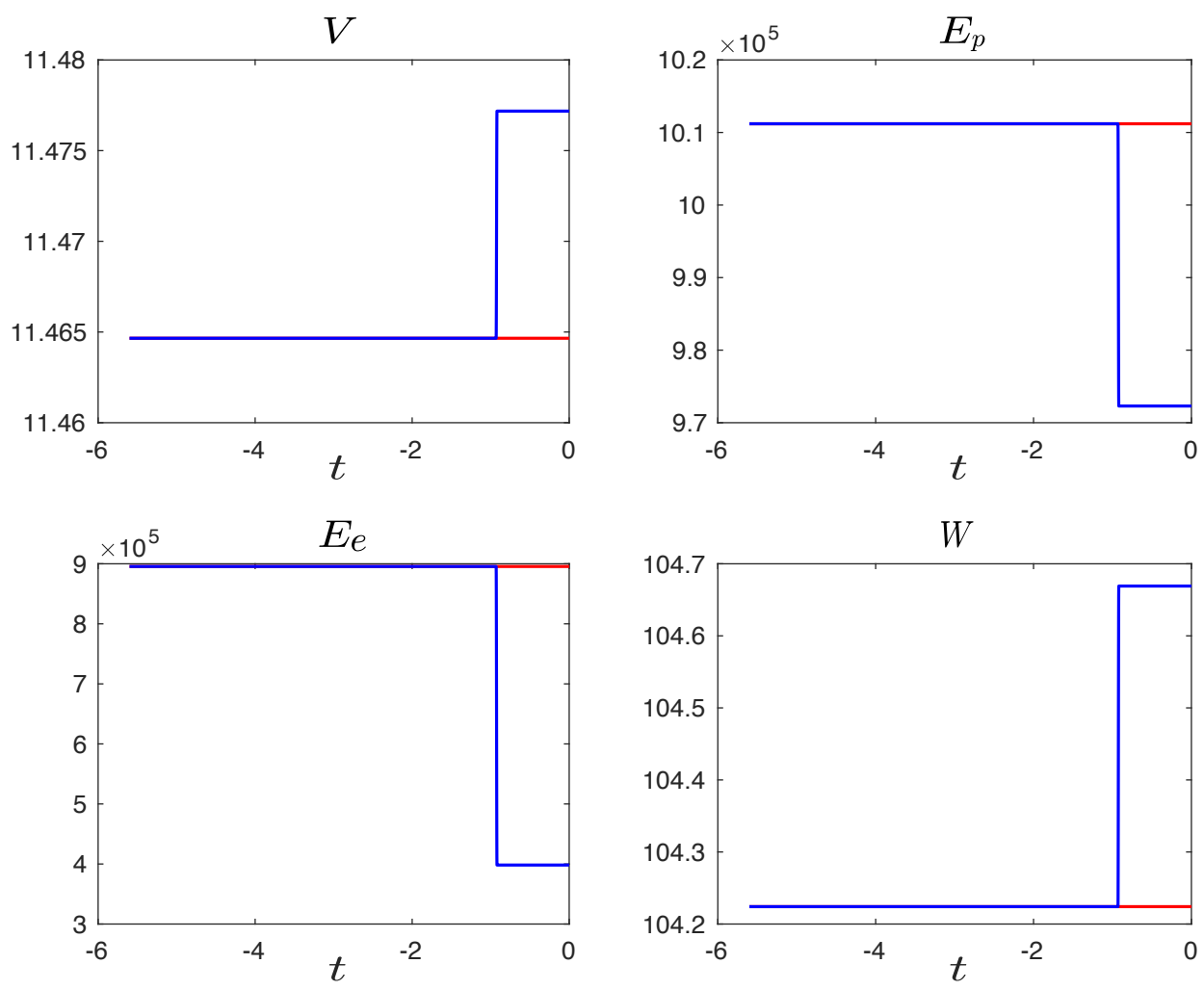

A
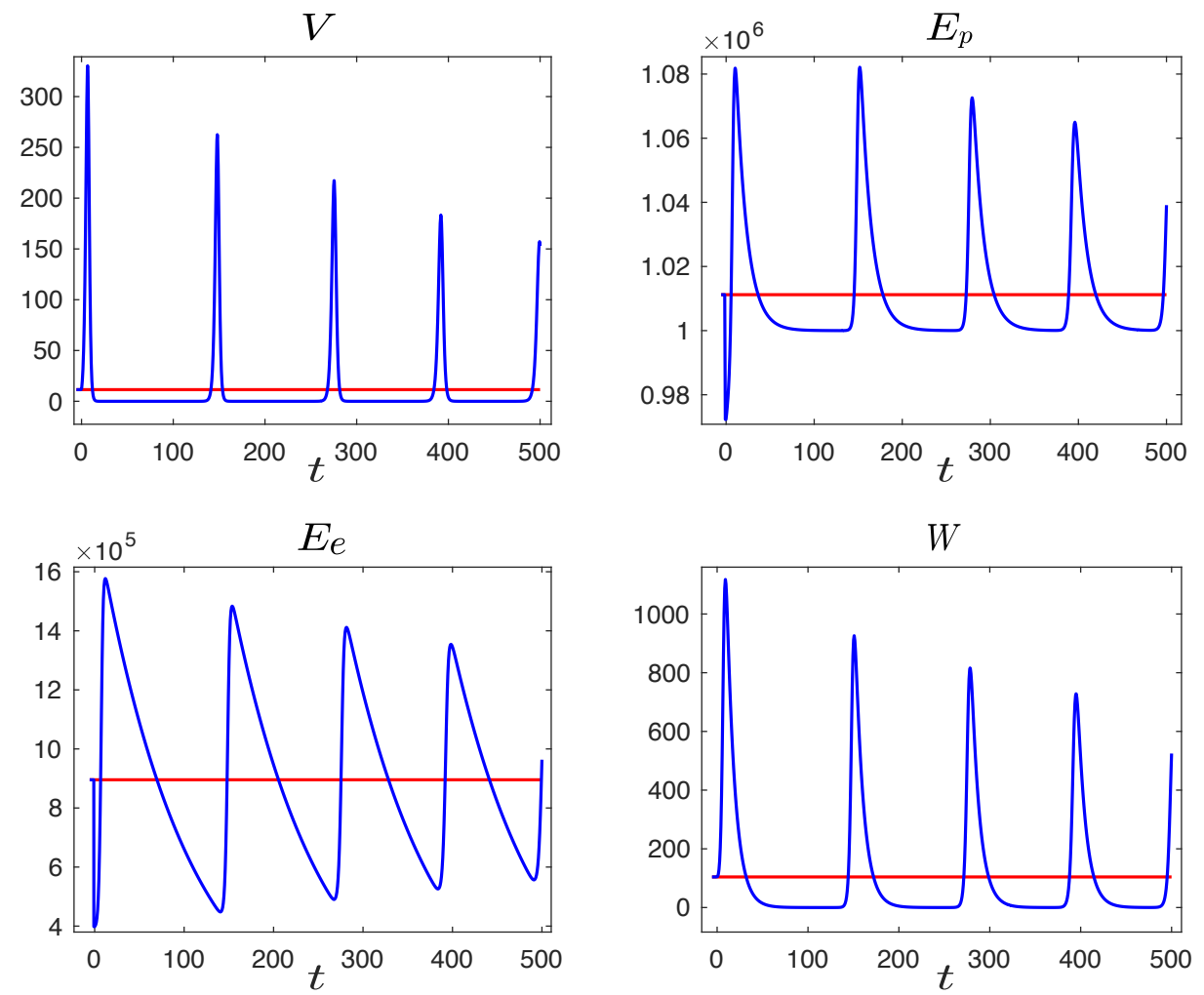

$\mathrm{B}$

Рис. 1: Начальное значение (А) и результат интегрирования возмущённого стационарного состояния $\bar{U}_{I}(\mathrm{~B})$ при $l=6$ и $\varepsilon=0.15$ 

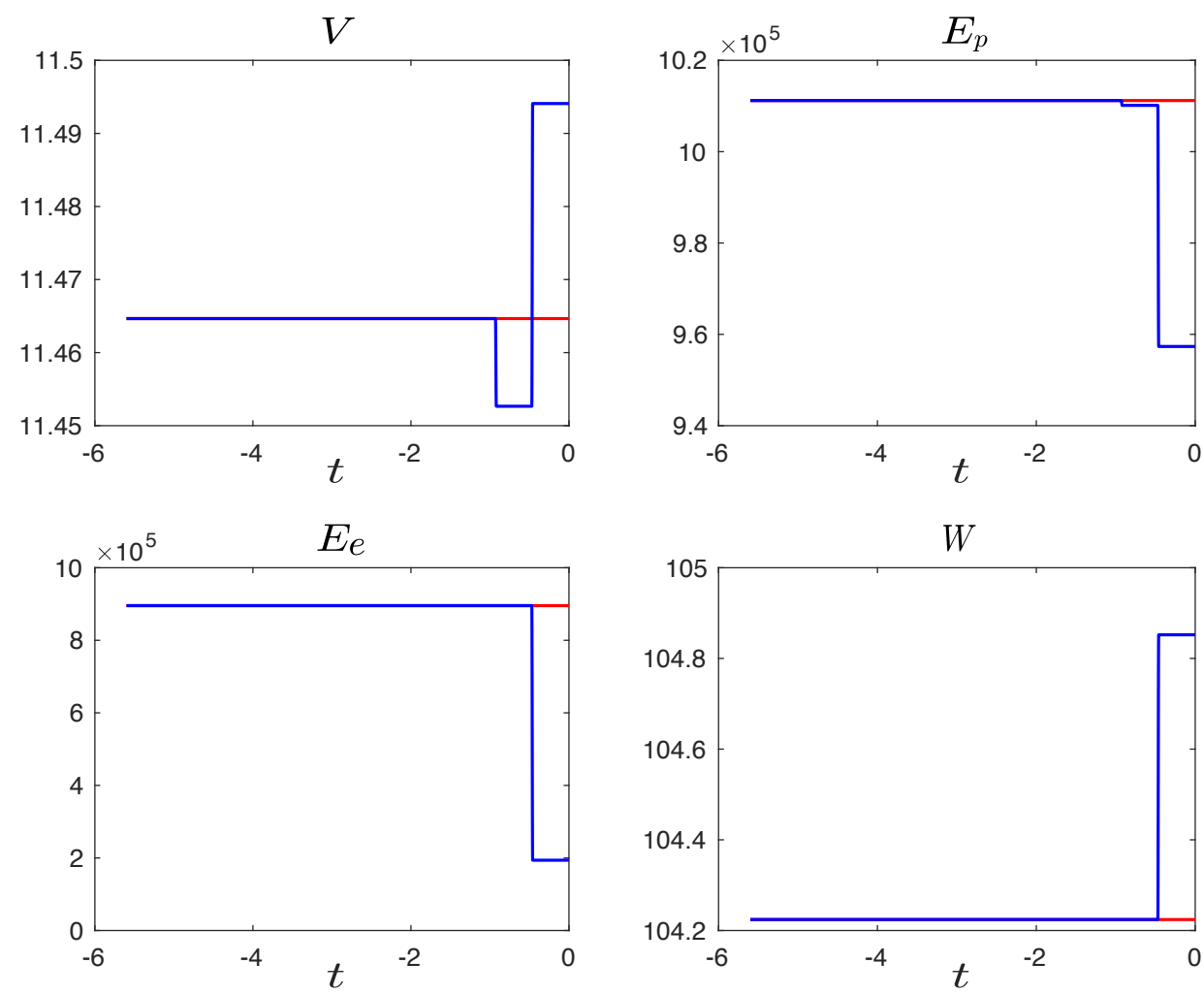

A
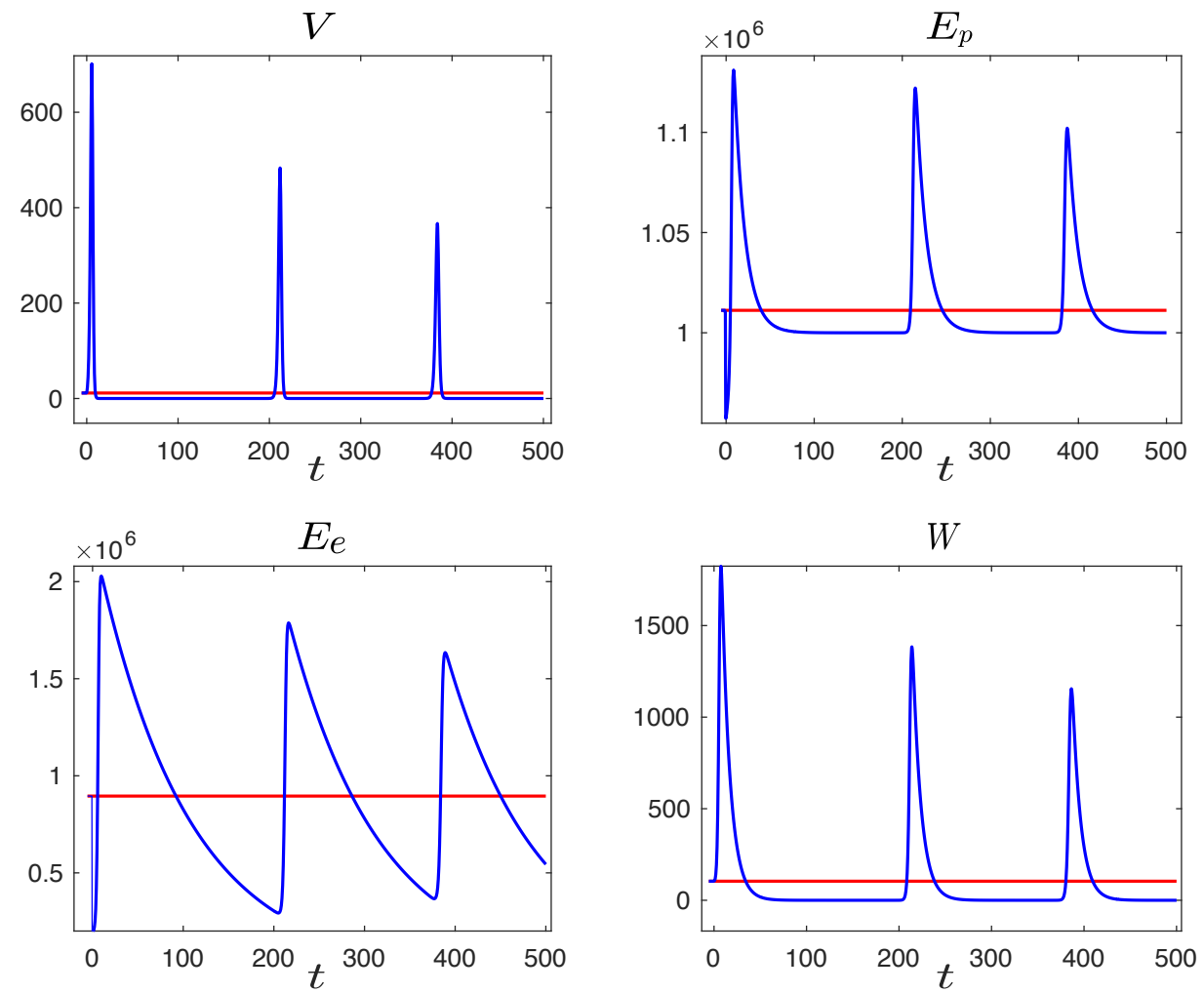

B

Рис. 2: Начальное значение $(\mathrm{A})$ и результат интегрирования возмущённого стационарного состояния $\bar{U}_{I}(\mathrm{~B})$ при $l=12$ и $\varepsilon=0.15$ 

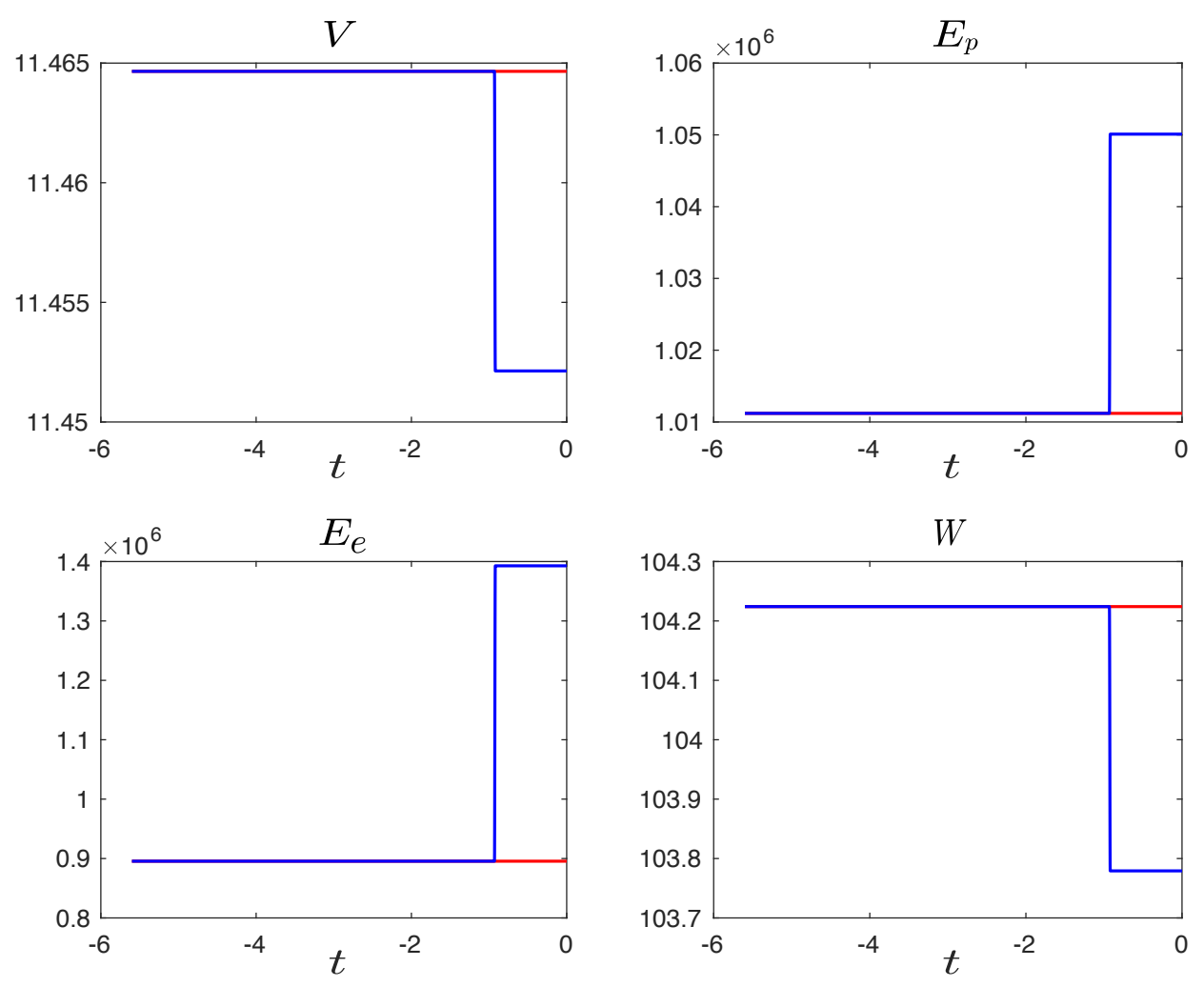

A
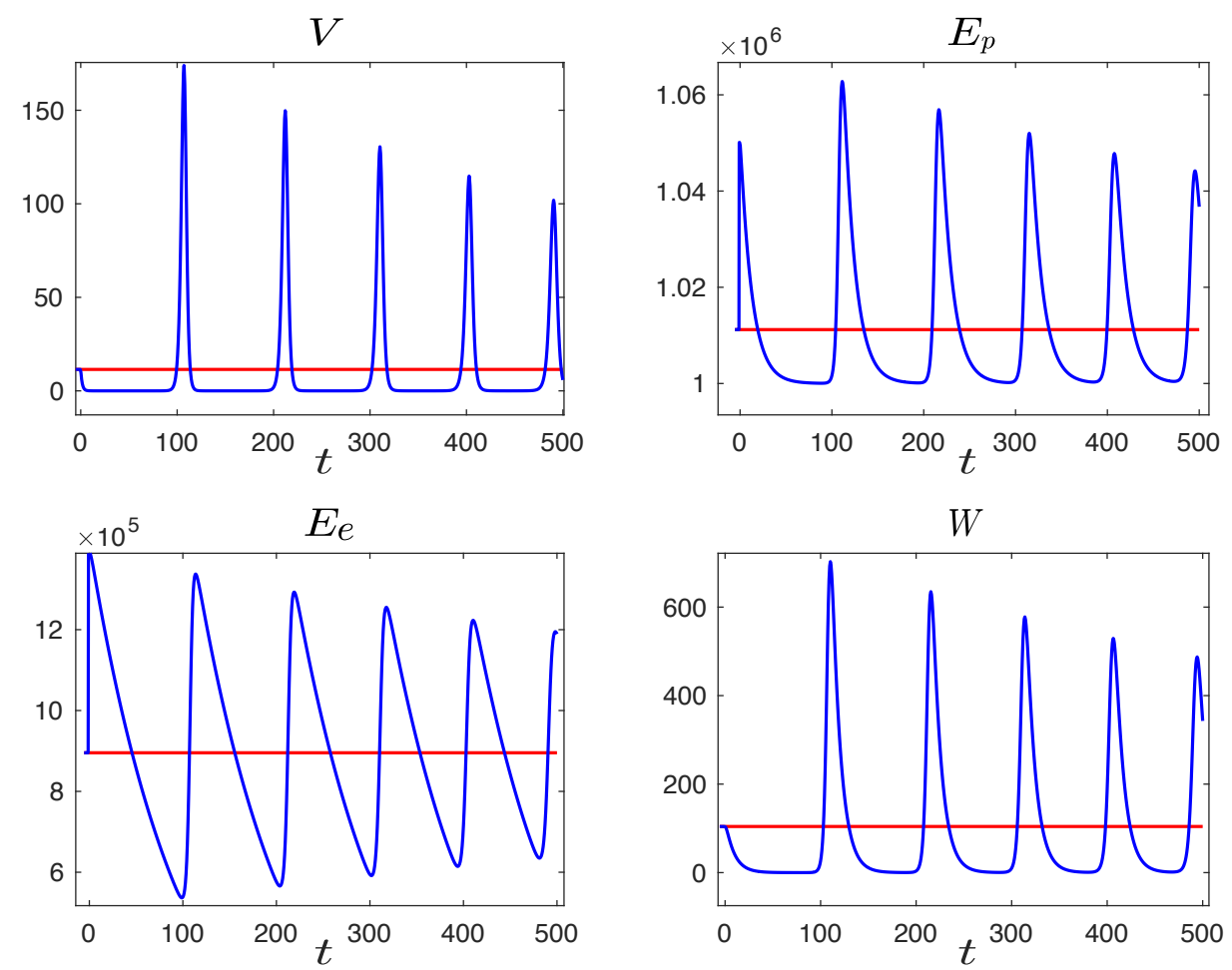

$\mathrm{B}$

Рис. 3: Начальное значение (A) и результат интегрирования возмущённого стационарного состояния $\bar{U}_{I}(\mathrm{~B})$ при $l=6$ и $\varepsilon=-0.15$ 

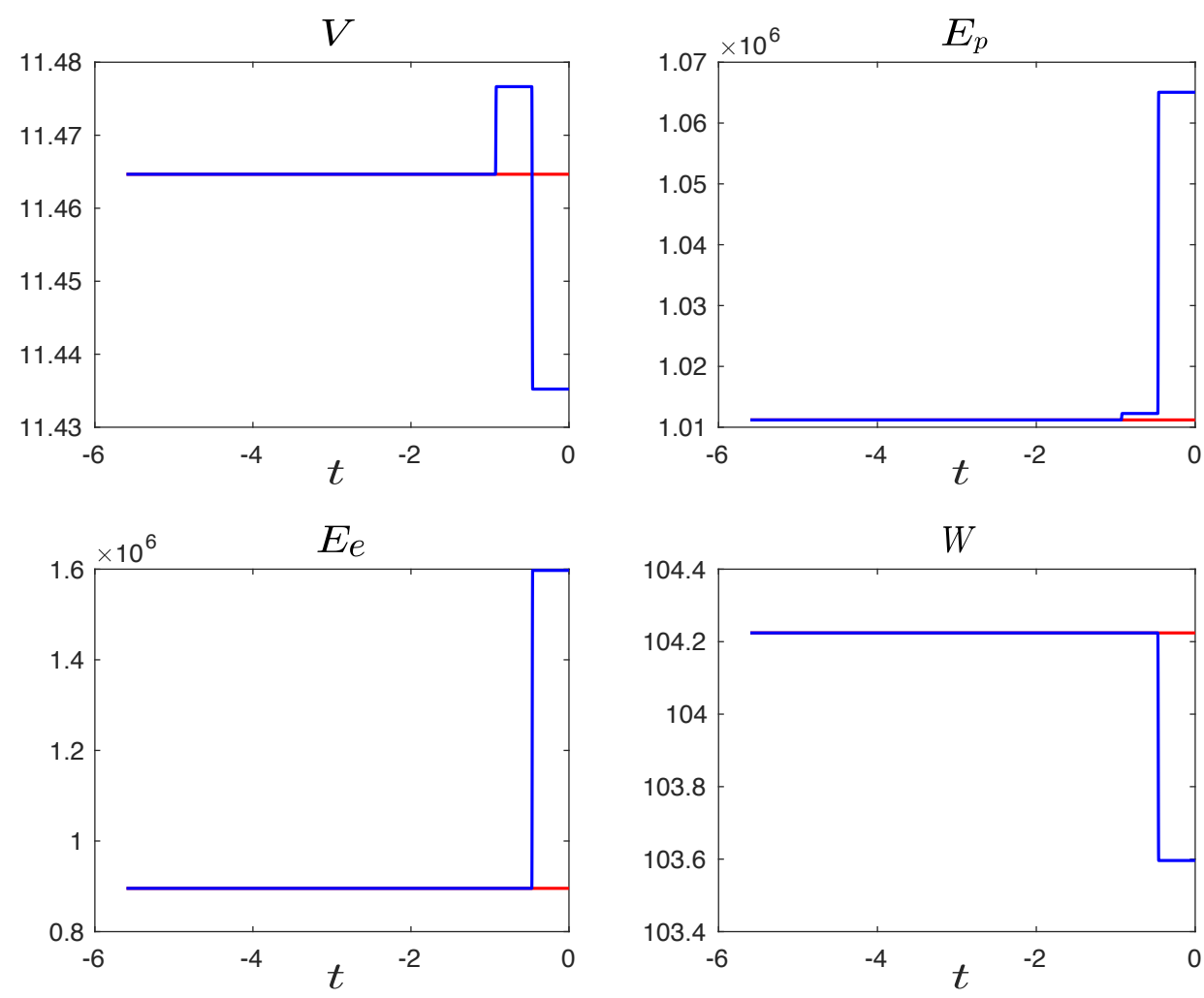

A
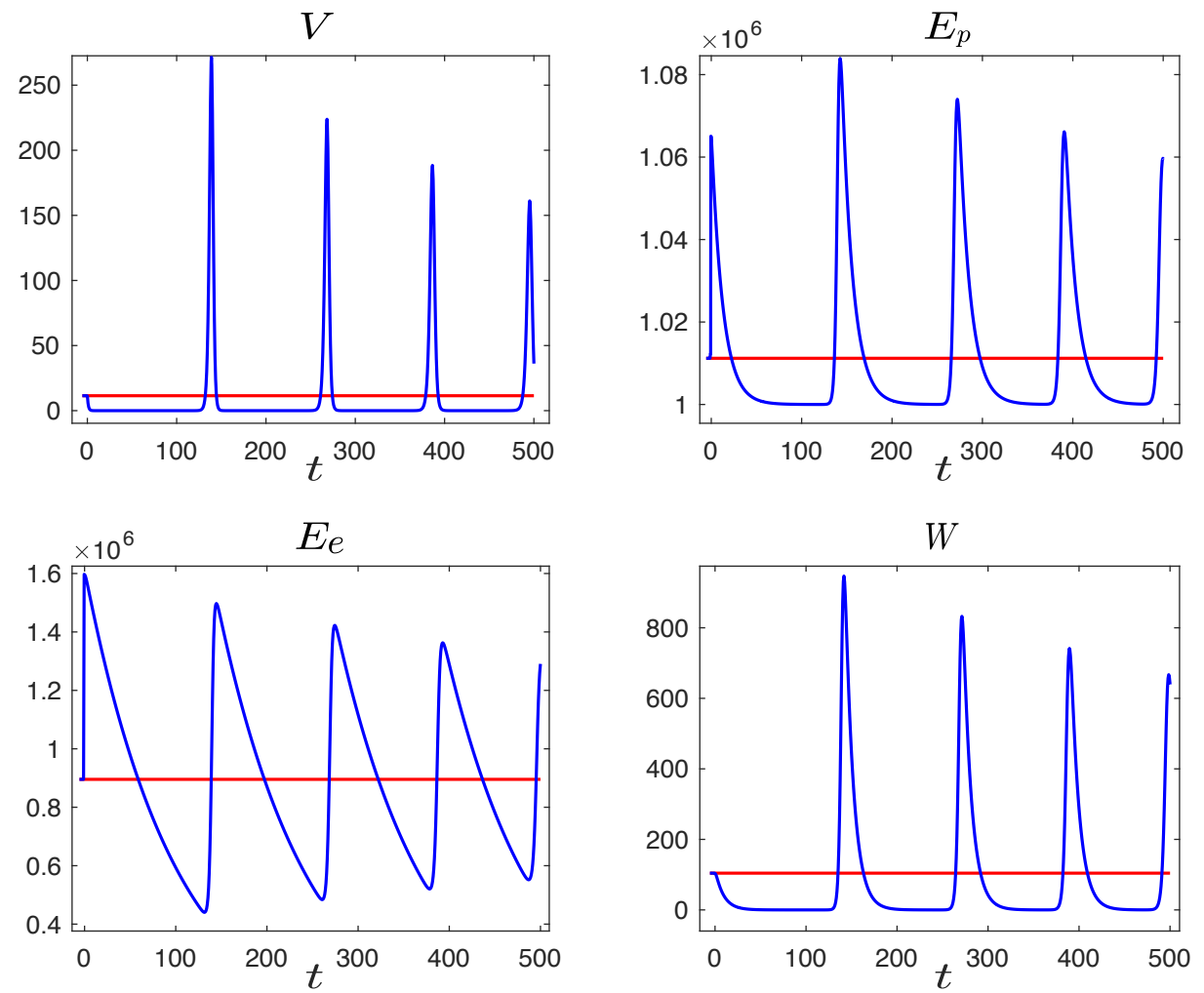

B

Рис. 4: Начальное значение $(\mathrm{A})$ и результат интегрирования возмущённого стационарного состояния $\bar{U}_{I}(\mathrm{~B})$ при $l=12$ и $\varepsilon=-0.15$ 

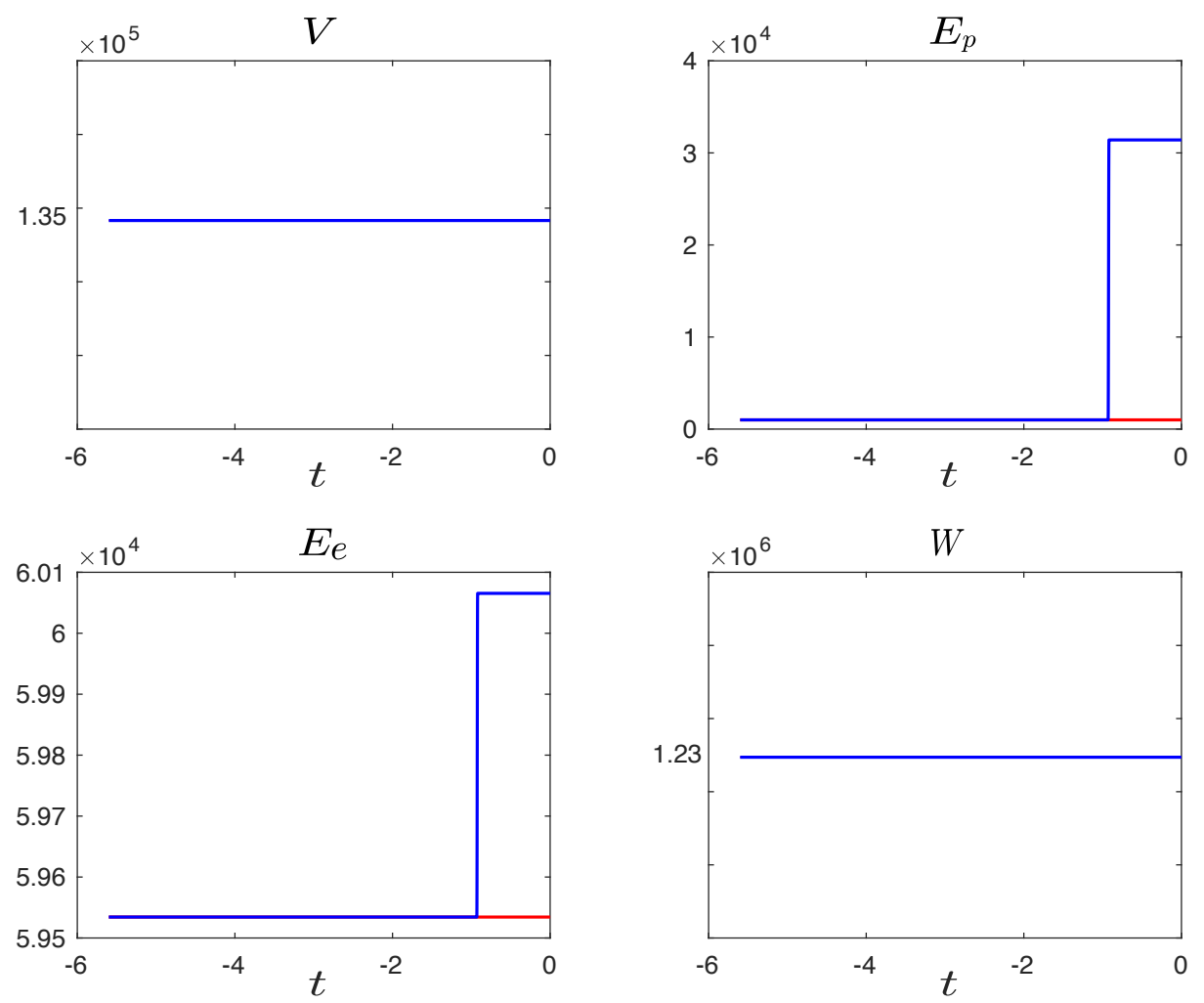

A
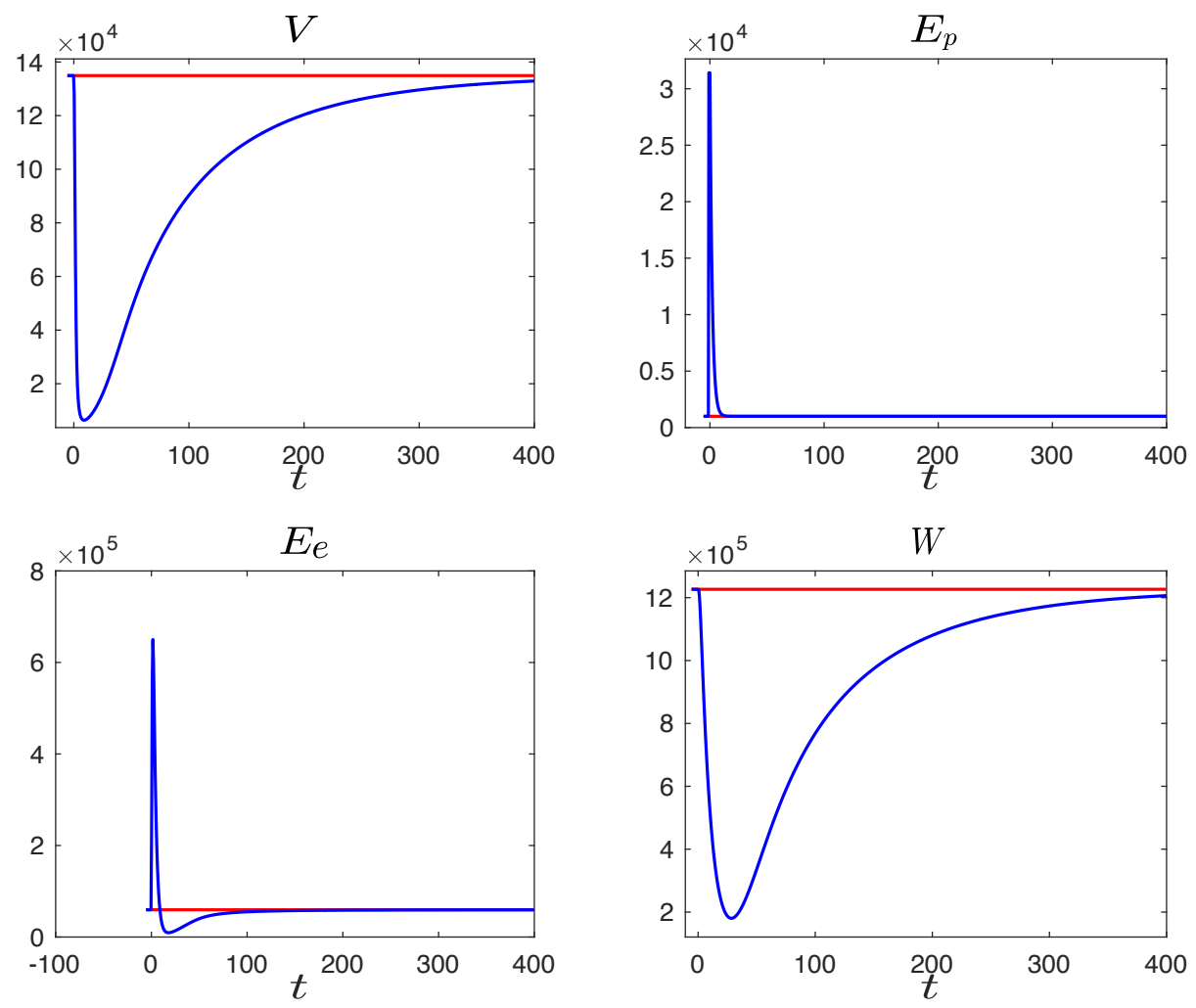

B

Рис. 5: Начальное значение $(\mathrm{A})$ и результат интегрирования возмущённого стационарного состояния $\bar{U}_{I I}(\mathrm{~B})$ при $l=6$ и $\varepsilon=-0.01$ 

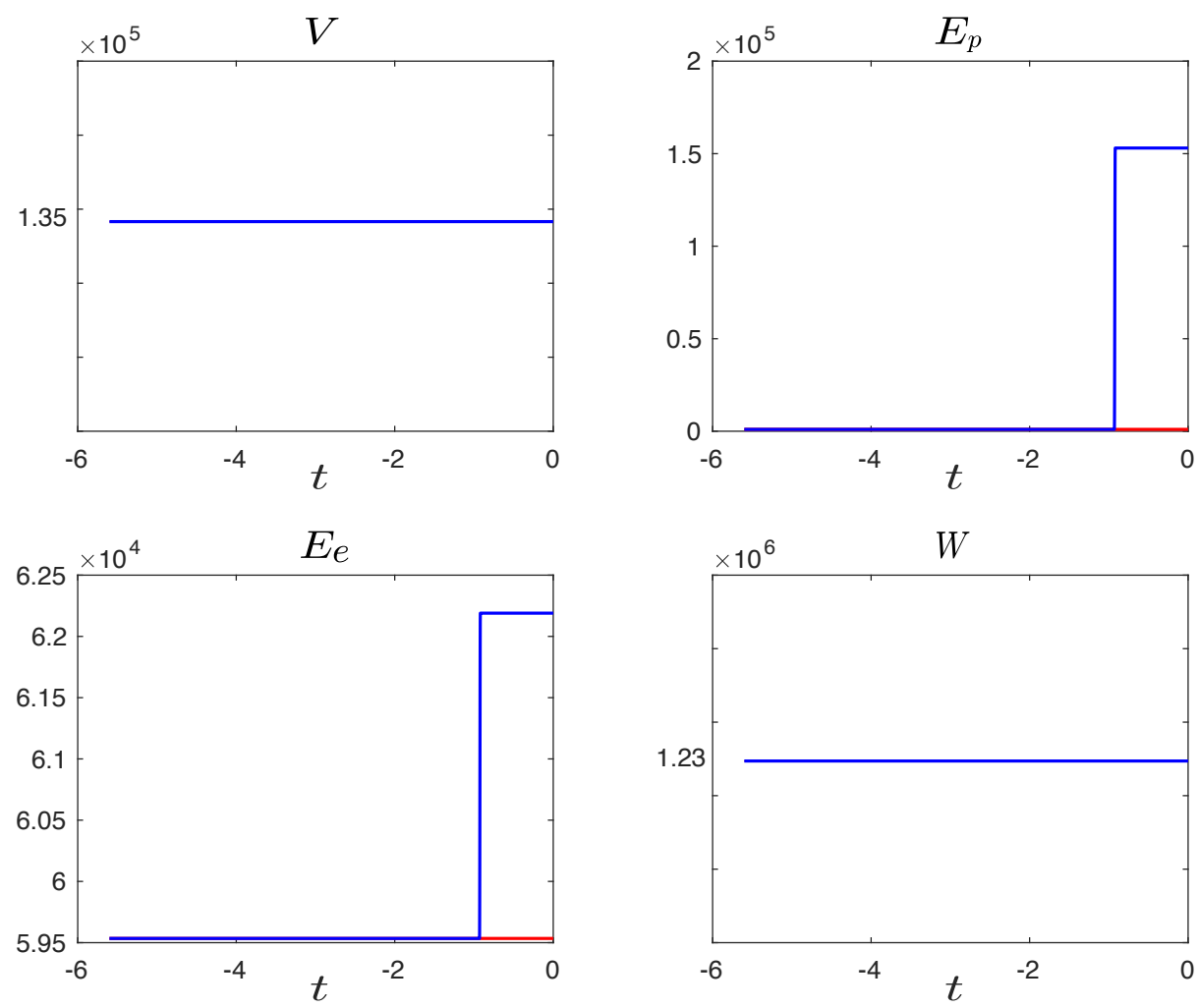

A
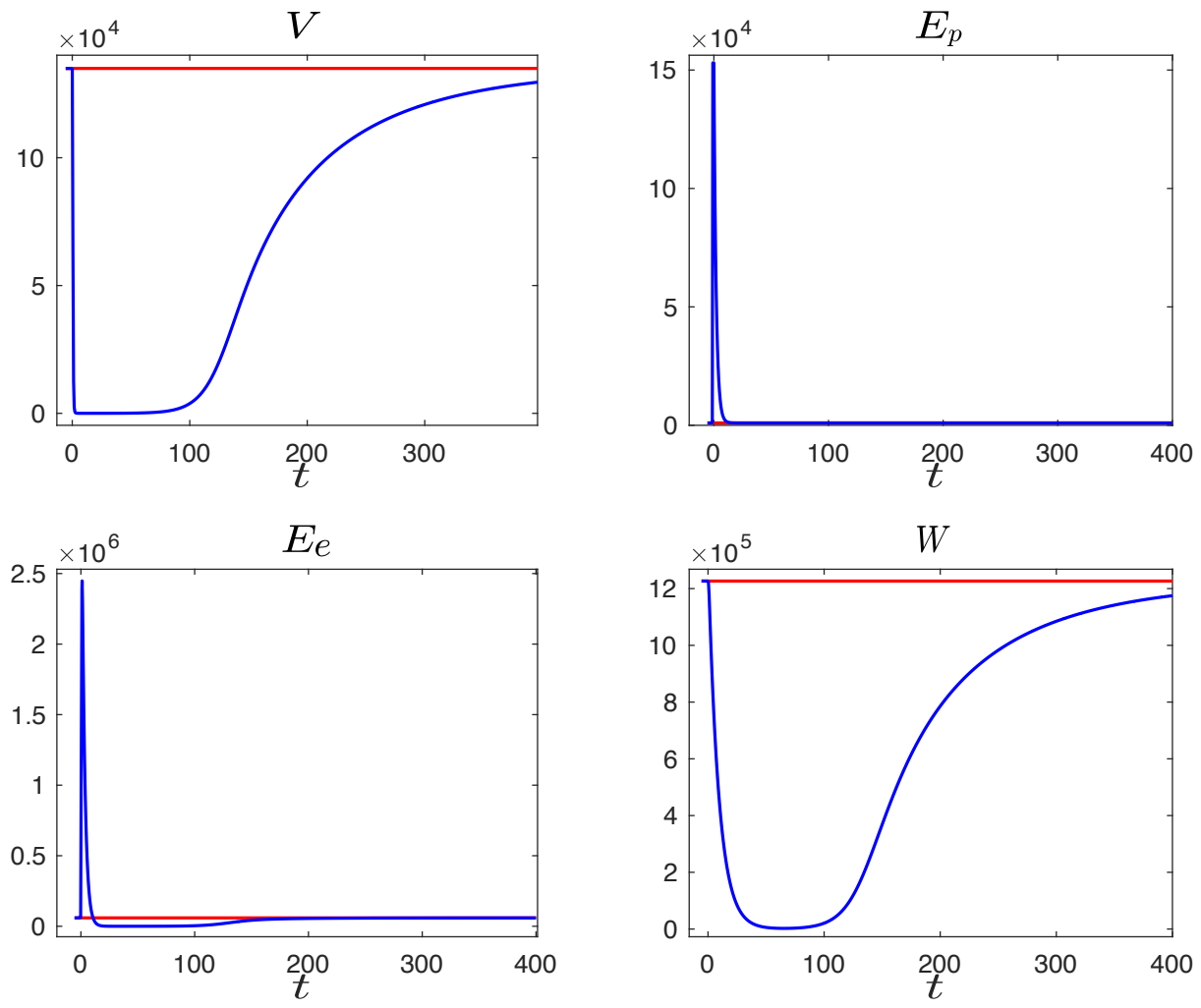

B

Рис. 6: Начальное значение (A) и результат интегрирования возмущённого стационарного состояния $\bar{U}_{I I}$ (В) при $l=6$ и $\varepsilon=-0.05$ 
Для первого состояния были независимо рассмотрены его возмущения с целью активации инфекционного процесса путем увеличения численности вирусной популяции с последующим удалением вирусного резервуара и с целью субклинической элиминации инфекции. Для второго состояния целью воздействия было снижение активности инфекционного процесса с последующим выведением вирусов из организма. Во всех рассмотренных случаях имела место значительная амплификация возмущений для рассмотренных вариантов воздействий на стационарное состояние системы. Тем самым, была показана принципиальная возможность использования специально подобранных оптимальных возмущений с малой начальной локальной нормой, оказывающих максимальное воздействие на динамику системы с точки зрения заданных критериев.

Важным и требующим дальнейших исследований остается вопрос о том, насколько значимыми являются возмущения малой амплитуды в компонентах стационарного состояния модели (являющиеся формальным решением оптимизационной задачи в ряде случаев) или, в более широком плане, вопрос единственности решения задачи построения оптимальных возмущений при заданных ограничениях на длительность возмущения. Выбор подпространства, в котором ищется возмущение, также должен быть связан с характеристиками реакции инфекционного- и иммунного процессов под действием антивирусных и иммуномодулирующих препаратов и будет конструктивно формализован в последующем.

Предложенная методология дает возможность развивать для задач клинической иммунологии подходы к лечению персистирующих и хронических инфекций, позволяющие при минимуме многокомпонентных лекарственных воздействий на организм добиться максимального лечебного эффекта. Практическая сложность решения данной задачи обусловлена необходимостью рассматривать многокомпонентные лечебные воздействия, фармакодинамику и фармакокинетику препаратов и параметризовать их эффекты в моделях в виде конкретных функциональных зависимостей. В свою очередь, это требует дальнейших междисциплинарных исследований, включая как вопросы построения биологически корректных математических моделей взаимодействия вирусов и организма человека, учитывающих иммунопатологические процессы развития инфекций, так и разработки робастных алгоритмов поиска оптимальных возмущений для многопараметрических моделей с большой размерностью фазового пространства.

В заключение, уместно привести слова классика современной иммунологии - Уильяма Пола [24]: «...иммунная система ставит сложные задачи перед исследователями в области биоматематики. Прежде всего - это вопросы количественного предсказания результатов воздействий на иммунную систему, в которых мы ожидаем непосредственного участия наших коллег 
математиков и специалистов по моделированию.» Данное положение является одной из важнейших мотиваций для дальнейших исследований, связанных с разработкой математических методов и моделей для приложений в иммунологии с целью построения эффективных подходов к лечению неблагоприятных форм течения инфекционных заболеваний.

\section{Список литературы}

[1] G. A. Bocharov. Modelling the dynamics of LCMV infection in mice: conventional and exhaustive CTL responses // J. Theor. Biol. 1998. Vol. 192, No. 3, P. 283-308.

[2] G. A. Bocharov, G. I. Marchuk, A. A. Romanyukha. Numerical solution by LMMs of stiff delay differential systems modelling an immune response Numerische Mathematik 1996. Vol. 73, No. 2, P. 131-148.

[3] A. V. Boiko, Y. M. Nechepurenko, M. Sadkane. Computing the maximum amplification of the solution norm of differential-algebraic systems // Comput. Math. Model. 2012. Vol. 23, No. 2, P. 216-227.

[4] A. V. Boiko, Y. M. Nechepurenko, M. Sadkane. Fast computation of optimal disturbances for duct flows with a given accuracy // Comput. Maths Math. Phys. 2010. Vol. 50, No. 11, P. 1914-1924.

[5] A. V. Boiko, A. V. Dovgal, G. R. Grek, V. V. Kozlov. Physics of Transitional Shear Flows: Instability and Laminar-Turbulent Transition in Incompressible Near-Wall Shear Layers. Berlin: Springer, 2011. 98 p.

[6] V. A. Chereshnev, G. Bocharov, S. Bazhan, B. Bachmetyev, I. Gainova, V. Likhoshvai, J. M. Argilaguet, J. P. Martinez, J. A. Rump, B. Mothe and others. Pathogenesis and treatment of HIV infection: the cellular, the immune system and the neuroendocrine systems perspective // Int. Rev. Immunol. 2013. Vol. 32, No. 3, P. 282-306.

[7] A. Ciurea, P. Klenerman, L. Hunziker, E. Horvath, B. Odermatt, A. F. Ochsenbein, H. Hengartner, R. M. Zinkernagel. Persistence of lymphocytic choriomeningitis virus at very low levels in immune mice // PNAS 1999. Vol. 96, No. 21, P. 11964-11969.

[8] A. Crawford, J. M. Angelosanto, C. Kao, T. A. Doering, P. M. Odorizzi, B. E. Barnett, E. J. Wherry. Molecular and transcriptional basis of CD4+ 
T cell dysfunction during chronic infection // Immunity 2014. Vol. 40, No. 2, P. 289-302.

[9] M. E. Csete, J. C. Doyle. Reverse engineering of biological complexity // Science 2002. Vol. 295, No. 5560, P. 1664-1669.

[10] G. H. Golub, C. F. Van Loan. Matrix Computations. Baltimore: The John Hopkins University Press, 1996.

[11] D. Gurdasani, L. Iles, D. G. Dillon, E. H. Young, A. D. Olson, V. Naranbhai, S. Fidler, E. Gkrania-Klotsas, F. A. Post, P. Kellam and others. A systematic review of definitions of extreme phenotypes of HIV control and progression // AIDS 2014. Vol. 28, No. 2, P. 149-162.

[12] E. Hairer, G. Wanner. Solving ordinary differential equations. Berlin: Springer-Verlag, 1996.

[13] S. J. Kent, J. C. Reece, J. Petravic, A. Martyushev, M. Kramski, R. De Rose, D. A. Cooper, A. D. Kelleher, S. Emery, P. U. Cameron and others. The search for an HIV cure: tackling latent infection // Lancet Infect. Dis. 2013. Vol.13, No. 7, P. 614-621.

[14] H. Kitano. Systems biology: a brief overview // Science 2002. Vol. 295, No. 5560, P. 1662-1664.

[15] H. Kitano. Biological robustness // Nat. Rev. Genet. 2004. Vol. 5, No. 11, P. 826-837.

[16] H. Kitano. Biological robustness in complex host-pathogen systems. Basel: Birkhäuser, 2007. P. 239-263.

[17] T. Luzyanina, K. Engelborghs, S. Ehl, P. Klenerman, G. Bocharov. Low level viral persistence after infection with LCMV: a quantitative insight through numerical bifurcation analysis // Math. Biosci. 2001. Vol. 173, No. 1, P. 1-23.

[18] G. I. Marchuk. Mathematical Modelling of Immune Response in Infectious Diseases/ Mathematics and Its Applications 395, Dordrecht: Kluwer, 1997.

[19] G. I. Marchuk. Mathematical models in immunology. New York: Optimization Software Inc. Publications Division, 1983.

[20] D. Moskophidis, F. Lechner, H. Pircher, R. M. Zinkernagel. Virus persistence in acutely infected immunocompetent mice by exhaustion of antiviral cytotoxic effector T cells // Nature 1993. Vol. 362, P. 758-758. 
[21] Y. M. Nechepurenko, M. Sadkane. Computing humps of the matrix exponential // J. Comput. Appl. Math. (2017 (to appear)).

[22] Y. M. Nechepurenko, M. Sadkane. A low-rank approximation for computing the matrix exponential norm // SIAM J. Matrix. Anal. Appl. 2011. Vol. 32, No. 2, P. 349-363.

[23] M. Nowak, R. M. May. Virus dynamics: mathematical principles of immunology and virology. Oxford: Oxford University Press, 2000.

[24] W. E. Paul. The Immune System-Complexity Exemplified // MMNP 2012. Vol. 7, No. 5, P. 4-6.

[25] A. S. Perelson, P. W. Nelson. Mathematical analysis of HIV-1 dynamics in vivo // SIAM Rev. 1999. Vol. 41, No. 1, P. 3-44.

[26] B. T. Polyak, P. S. Shcherbakov, M. V. Khlebnikov. Control of linear systems subjected to exogenous disturbances: the linear matrix inequality technique. Moscow: LENAND, 2014. 